\title{
Aplicación Clínica de Modelos Terapéuticos No Mediacionales en un Caso de Trastorno Mixto del Afecto ${ }^{1}$
}

\author{
Clinical Implementation of Non Mediational Therapeutic Models \\ in a Mixed Affective Disorder Case
}

\author{
Mónica Ma. Novoa Gómez \\ Diana Guzmán Gutiérrez \\ Pontificia Universidad Javeriana, Colombia
}

(Rec: 28 enero 2008 Acep: 05 noviembre 2008)

\begin{abstract}
Resumen
El presente artículo expone un estudio de caso siguiendo la formulación analítica conductual y los componentes y principios de la intervención conductual planteados por la Terapia de Aceptación y Compromiso y la Psicoterapia Analítica Funcional, que retoma la importancia de la conducta verbal y sus características al momento de formular e intervenir en casos como el de un joven que acude a consulta psicológica con diagnóstico de trastorno depresivo y ansiedad por déficit en habilidades sociales, como en el presente caso en particular. Los resultados señalan la utilidad terapéutica de las estrategias empleadas, especialmente en el componente de compromiso y autocontrol que el consultante desarrolló. Discute además la pertinencia de abordar el caso clínico con énfasis en procesos cognitivo-verbales, desde modelos terapéuticos no mediacionales.
\end{abstract}

Palabras clave: Psicoterapia analítica funcional (PAF), terapia de aceptación y compromiso (ACT, ) depresión, ansiedad por déficit en habilidades sociales.

\begin{abstract}
This paper presents a case study following the analytical formulation and behavioral components and principles of the behavioral intervention posed by Acceptance and Commitment Therapy and Functional Analytic Psychotherapy, which takes up the importance of verbal and their characteristics when formulating and intervening on cases such as the one of a young man who goes to psychological consultation with a diagnosis of depressive disorder and anxiety for deficits in social skills, as in this particular case. The results indicate the usefulness of the therapeutic strategies employed, particularly in the component of commitment and selfcontrol that the consultant developed. The relevance of addressing the case with an emphasis on cognitive processes like verbal processes is discussed, from the use of non mediational therapeutic models.
\end{abstract}

Key words: Functional analytic psychotherapy (FAP), acceptance and commitment therapy (ACT), depression, social skills deficit. 


\section{Introducción}

Bajo el término depresión se han agrupado una serie de características conductuales, que en general recogen sentimientos de profunda tristeza, melancolía, infelicidad, sentirse miserable o derrumbado y una pérdida de interés en actividades que anteriormente la persona consideraba como divertidas o placenteras. Puede acompañarse de una variedad de alteraciones de carácter psicofisiológico, emocionales (hiperreactividad o hipoactividad emocional) y sociales (retraimiento).

Autores como Scholten (2003) distinguen la depresión de episodios de melancolía, en función de la duración de los mismos, definiendo la depresión como un trastorno del estado de ánimo que puede durar semanas, meses o años, en el que los sentimientos de tristeza, pérdida, ira o frustración interfieren con la vida diaria.

Ha sido ampliamente señalado que este tipo de trastornos del afecto raramente se recuperan sin tratamiento. A su vez, el DSM-IVTR lo describe como un trastorno del afecto que requiere de al menos un episodio depresivo mayor, caracterizado por estado de ánimo depresivo, disminución del interés por el placer o las actividades, pérdida de peso $\mathrm{y} / \mathrm{o}$ cambios en el apetito, insomnio o hipersomnia, enlentecimiento o agitación psicomotora, fatiga o pérdida de energía, sentimientos de inutilidad o de culpa, dificultad para pensar, concentrarse y tomar decisiones y pensamientos recurrentes de muerte (Gómez \& Roa, 2002).

Dada la prevalencia de este tipo de trastornos en la población colombiana, reportada en el último Estudio Nacional de Salud Mental (2003) $)^{2}$, se presenta como fundamental la indagación científica sobre la efectividad terapéutica y la coherencia de un modelo explicativo, sin embargo, esto se constituye aún en una tarea sin concluir.

Los modelos explicativos de este tipo de trastorno son abundantes en la literatura clínica tanto psiquiátrica como psicológica y pueden resumirse en dos ejes, según el énfasis dado; los modelos que dan predominio a la sumatoria de factores genéticos y estructurales a nivel orgánico, así como aquellos que dan un alto nivel de importancia a determinados procesos cognoscitivos, afectivos y sociales.

Los factores biológicos implicados están relacionados principalmente con una disminución de las monoaminas biógenas como la serotonina, noradrenalina y dopamina a nivel del sistema nervioso central por la inhibición de la recaptación de la serotonina. Además, se presentan alteraciones neuroendocrinas, pues la depresión se asocia con signos de alteración hipotalámica que se manifiesta en una secreción excesiva de la hormona corticotropina $(\mathrm{ACTH})$ por la hipófisis, induciendo aumento de la secreción de cortisol por la corteza suprarrenal.

\footnotetext{
Ministerio de la Protección Social República de Colombia (2003) Un panorama Nacional de la Salud y la Enfermedad mental en Colombia. Informe Preliminar.
}

Brown, Varghese y McEwen (2004) señalan que el exceso de cortisol en el trastorno depresivo mayor puede estar causado por la activación en las horas de la tarde del eje hipotálamo-pituitario-adrenal (HPA), evidencia que no se encuentra en las personas que no presentan la sintomatología depresiva.

Los estudios han demostrado numerosas anomalías en el eje HPA en los pacientes deprimidos (Dinan, 2001). Igualmente se producen modificaciones neuroanatómicas como se ha visto en los estudios de tomografía por emisión de positrones (PET) y de resonancia magnética funcional (RMf) que han determinado una posible alteración anatómica en la corteza prefrontal ventral a la rodilla del cuerpo calloso, alterada en los casos de depresión unipolar. Durante la fase depresiva de la enfermedad, la actividad en esta región disminuye (Montes, 2004).

Mediante técnicas de neuroimagen estructural (tomografía computarizada y RM) se han investigado posibles anomalías cerebrales relacionadas con la depresión. Igualmente los estudios de neuroimagen funcional (PET y SPECT) confirman que existen disfunciones metabólicas en el lóbulo frontal (Pérez-Gómez, Junqué, Mercader \& Berenguer, 2000). Frodl, Meisenzahl, Zetzsche, Born, Groll y Jager (2002) citados por Brown, Varghese y McEwen (2004) encontraron que durante el primer episodio de depresión se presentaba una disminución del volumen del hipocampo, lo cual representa un factor de riesgo de la depresión.

Desde la perspectiva psicológica, algunos modelos que relacionan la depresión con el estilo cognitivo, explican los factores de mantenimiento del trastorno según el tipo de atribución de la causalidad que una persona haga de un evento traumático e incontrolable que determinará la manifestación y la extensión de la depresión (Burger, 1984).

Han sido desarrollados en esta línea los modelos que se basaron en el modelo de indefensión aprendida, planteada por Seligman en 1974 (Abramson, Seligman \& Teasdale, 1978; Seligman, Abramson \& Semmel, 1979) que explican el patrón depresivo según las atribuciones de causalidad que el sujeto hace de las situaciones de la vida cotidiana, pero especialmente de aquellas en las cuales no obtiene los resultados esperados.

Las atribuciones podrían ser: internas/externas que se relacionan con la culpa, estables/inestables, asociadas con la cronicidad y globales/específicas, que tendrían que ver con la generalización.

Los modelos de depresión de la indefensión aprendida señalan que la reacción de un individuo a las experiencias donde el control personal está ausente, depende de qué tan importante sea para la persona controlar dicha situación. Según el modelo cognitivo, mientras más importante el individuo considere controlar el evento, más grande será el esfuerzo de incrementar el control y más severa será la reacción de inutilidad (Burger, 1984). 
Los estudios se han enfocado comúnmente en los predictores clínicos y cronicidad de la depresión, muy pocos se han centrado en explorar el por qué algunos individuos permanecen con una depresión crónica mientras que otros experimentan un curso episódico.

Lara y Klein (1999) plantean tres perspectivas psicosociales para explicar la persistencia de la depresión.

La primera de ellas tiene sus raíces en la teoría cognitiva y sostiene que se ha establecido una relación recíproca entre los síntomas depresivos y la percepción y la respuesta del individuo a esos síntomas, lo cual mantiene y prolonga la duración de los episodios de depresión. Teasdale (1985 citado por Lara \& Klein, 1999) plantea que los síntomas depresivos en un episodio de depresión, activan los recuerdos y constructos negativos que son después utilizados para interpretar nuevos eventos y de ésta manera se mantiene la depresión en un círculo vicioso. Éste ciclo negativo de pensamiento generará una condición depresiva persistente.

La segunda perspectiva surge de la teoría interpersonal y propone que los comportamientos de la persona deprimida crean un ambiente social negativo, que perpetúa los episodios depresivos. Lewinsohn (1974 citado por Lara \& Klein, 1999) es uno de los autores relacionados con esta perspectiva, quien sostiene que la ausencia de habilidades sociales en la depresión y la disminución en la habilidad para disfrutar los eventos positivos, reduce la posibilidad de experimentar un reforzamiento positivo del ambiente; y además, que los síntomas depresivos son mantenidos a través de las contingencias ambientales sociales como la compasión y la preocupación.

Quiñones y Sandoval (1996) citando a Coyne (1989), afirman, en la misma línea de los autores mencionados en esta segunda perspectiva, que el depresivo en sus etapas iniciales atrae la atención de sus semejantes, esto mantiene el brote de los pacientes hasta que es aislado completamente de su medio social. De acuerdo con este enunciado, se ha logrado reconocer la importancia del medio social y se ha hecho posible diferenciar el medio social de un paciente depresivo y el de un paciente normal, caracterizado por redes de apoyo menores, ser menos aceptados socialmente, poseer un nivel menor de habilidades sociales y ser o haber sido parte de familias disgregadas (Quiñones \& Sandoval, 1996).

Y la tercera perspectiva que emerge recientemente, se centra en que los episodios de adversidad en la niñez como el abuso o una paternidad/maternidad pobres, hacen que una persona sea mucho más susceptible a la aparición de una depresión duradera y crónica. El bajo cuidado parental y la alta sobreprotección han sido las características más comunes en los individuos con depresión.

Lara y Klein (1999) aclaran que estas tres perspectivas de persistencia y mantenimiento de la depresión aunque se conciben separadas se deben tratar de interrelacionar. Por ejemplo, los patrones particulares de pensamiento y las dificultades interpersonales pueden mediar los efectos de las experiencias aversivas tempranas en el desarrollo de la depresión crónica. Igualmente, los patrones de pensamientos negativos pueden ser una consecuencia de la adversidad en la niñez y conllevar a la persistencia de la depresión. Además el abuso en la edad infantil o la indiferencia de los padres pueden enseñar al niño que no tiene a nadie a quien acudir para que le brinde ayuda para resolver sus problemas lo cual puede llevar a que se presenten habilidades de afrontamiento pasivas.

De forma complementaria, desde las teorías conductuales clásicas, la depresión se entiende como el resultado en la disminución de la tasa de refuerzo y calidad del mismo, considerándose como un factor desencadenante para el trastorno. Esta tasa de refuerzo disminuida interactúa con pobres repertorios sociales para determinar los comportamientos depresivos, generando una disminución de la motivación hacía actividades anteriormente valoradas (Quiñones \& Sandoval, 1996).

Kanter, Cautilli, Busch, Baruch (2005) sostienen que los modelos conductuales tienen diferentes explicaciones para la depresión y que pueden relacionarse con un tratamiento conductual adecuado que involucra diferentes factores. Los factores relacionados con estos modelos enfatizan en 1) la depresión como una función de la deprivación de refuerzo positivo, en términos de una ausencia de respuesta contingente al reforzamiento del comportamiento, 2) relacionada con el control aversivo y el castigo, 3) el comportamiento depresivo es un comportamiento operante sujeto a y mantenido por el 4) reforzamiento positivo y 5) el reforzamiento negativo.

El primer factor, relacionado con la deprivación de reforzamiento supone que la ausencia o la reducción del reforzamiento positivo producen depresión.

Kanter y cols. (2005) relacionan la depresión con varios principios conductuales que incluyen la pérdida de refuerzo, erosión del reforzamiento y déficit en habilidades. Las reducciones de los niveles de reforzamiento pueden producir reducciones en las respuestas.

Las investigaciones de Kanner, Coyne, Schaefer y Lazarus, 1981 y Paykel, 1982 (citados por Kanter et al., 2005) indican que la mayoría de las personas deprimidas no reportan que una gran pérdida haya precipitado la depresión, por el contrario, la acumulación de pequeños y múltiples eventos estresantes a lo largo del tiempo son los que pueden predecir la depresión en una proporción mayor de individuos.

Por su parte, la erosión del reforzamiento refiere que se presentan procesos como la saciedad y habituación donde los estímulos reforzantes previos se vuelven menos reforzantes con el tiempo. Por último, Kanter et al. (2005) resaltan que el déficit en habilidades sociales en la depresión ha sido ampliamente investigado. Inicialmente se planteaba 
que el individuo debería tener en su repertorio las habilidades necesarias para obtener el refuerzo social y si estas habilidades eran deficientes los niveles de refuerzo social se reducirían y surgiría la depresión. Por otra parte, se plantea que las alteraciones en el funcionamiento interpersonal pueden ser un producto de un déficit en las habilidades sociales, convirtiéndose en precipitantes y consecuentes de la depresión. Aunque aporta mucho a la explicación, este modelo no puede explicar por completo el fenómeno de la depresión.

Otra visión menciona las habilidades de afrontamiento, donde las personas deben tener en su repertorio las destrezas necesarias no sólo para obtener refuerzo social sino también para ajustarse exitosamente a los eventos adversos y estresantes que se presenten en su vida. Este modelo también plantea que además de las habilidades sociales, de solución de problemas y regulación emocional se consideran importantes habilidades de afrontamiento que son relevantes en los estados de depresión (Kanter et al., 2005).

Tradicionalmente la visión conductual plantea que la depresión es una función del reforzamiento positivo, mientras que la ansiedad es una función del reforzamiento negativo y se ha hablado muy poco del castigo.

El segundo factor -control aversivo y castigo- explica que una historia de control aversivo excesivo puede producir un cúmulo de eventos que se relacionan con la depresión, la ansiedad o ambos. Se ha visto en algunos estudios que los niños y adolescentes deprimidos describen sus contextos familiares como hostiles.

El tercer factor se refiere a la pérdida del comportamiento operante efectivo, donde se menciona que el castigo generalizado resulta de gran importancia pues es común que las personas con depresión crónica reporten historias de su niñez de castigo prolongado del que no podían escapar. Algunos experimentos con humanos han mostrado que la incapacidad para realizar comportamientos efectivos a lo largo del tiempo, conlleva a la extinción de clases operantes de conducta. Por ejemplo, en un experimento donde se exponía a los participantes a ruidos de los que no se podían escapar, posteriormente se tornaban mucho más lentos para escapar del ruido cuando ello era posible (Kanter et al. 2005).

Los comportamientos de malestar, como quejarse u otras expresiones de preocupación, pueden considerarse como una clase de comportamientos que históricamente han sido reforzados por la comunidad social y verbal, lo cual está relacionado con el reforzamiento positivo del comportamiento depresivo (cuarto factor). Se ha encontrado que los niveles de las conductas de dolor verbales y no verbales de los pacientes con dolor crónico se asocian con un aumento en los niveles de las respuestas de atención de los acompañantes, sugiriendo que este comportamiento de los familiares o amigos puede mantener altos niveles de conductas de dolor a través del reforzamiento positivo.
Finalmente, el quinto factor relacionado con el reforzamiento negativo del comportamiento depresivo establece que el comportamiento depresivo puede tener la función de evitar o escapar. Por ejemplo, evitar el contacto con la comunidad social o dormir en exceso, ayudan a evitar la exposición a los estímulos ambientales que elicitan los pensamientos o sentimientos aversivos y de esta manera mantener el comportamiento depresivo y reducir el contacto con los reforzadores potenciales (Kanter et al., 2005).

Desde una perspectiva más reciente, la tercera generación de terapias conductuales (Hayes, 2001) aunque mantienen técnicas de las anteriores, se orientan en una dirección distinta.

En su conjunto, estas terapias, proponen elementos como por ejemplo, las terapias de corte existencial y experiencial ACT, cuyas siglas refieren el ACTUAR en dirección valiosa, aceptando -estando dispuesto a tener, dándose permiso para tener...- los eventos privados que conlleven malestar y surjan al actuar en la dirección elegida. Esta terapia apuesta por un cambio a un nivel diferente, ya que no se centra en el cambio (control) de los contenidos cognitivos o eventos privados, sino en la alteración del contexto relacional y la función que asumen en ese determinado contexto, en el que estos resultan problemáticos y en potenciar la exposición a los mismos. ACT es probablemente la más completa de estas nuevas terapias contextuales por las características que la definen: (1) parte de un marco global de referencia sobre las ventajas y desventajas de la condición humana, (2) mantiene una filosofia contextual-funcional, (3) es coherente con un modelo funcional sobre la cognición y el lenguaje, lo cual hace referencia a la Teoría del Marco Relacional y (4) sustenta una perspectiva nueva de la psicopatología en la que resulta central el concepto de evitación experiencial destructiva.

Desde esta nueva visión, se entiende que la conexión entre investigación básica, psicopatología y métodos clínicos es esencial para progresar en la prevención y la alteración de los trastornos psicológicos (Dougher, 2000; Hayes, 2001; Wilson \& Luciano, 2002). Estos modelos retoman como concepto fundamental, el planteamiento de la conducta gobernada por reglas planteado por Skinner (1953, 1957, 1966, 1974) aunque hacen énfasis en el papel del oyente mientras que Skinner hacia énfasis en el papel del hablante. Las reglas, desde esta postura, se entienden como estímulos discriminativos verbales que establecen la conducta y especifican las relaciones contingentes que funcionan como antecedentes de la conducta operante.

Las habilidades del individuo de generar reglas se ha aprendido a través de una historia de reforzamiento social por el seguimiento de reglas. Por lo cual se hablaría de que hay unas instrucciones establecidas culturalmente en las sociedades desarrolladas en la necesidad de hacerse cargo de las características de ser organismos verbales. Esto es, de manera dominante se propugna a través de textos y medios 
de divulgación que lo ideal para vivir es "sentirse bien", haciéndolo equivalente a (1) promover las sensaciones, los pensamientos y los recuerdos con funciones positivas y (2) la lucha contra, el rechazo o supresión de los eventos cognitivos negativos tan pronto surjan.

Las reglas que se ofrecen "inocentemente" por doquier son fórmulas para vivir que nos dicen: "la angustia, los recuerdos penosos, la tristeza, la baja autoestima, las sensaciones desagradables, el dolor, entre otras con características similares. Son barreras para vivir". Lo que aconsejan esas fórmulas es "evita tanto como puedas toda esa miseria y suprímela en cuanto aparezca, para que puedas hacer lo que te importa en la vida..." "Sentir cosas que señalan malestar es malo, solo es bueno sentir cosas que te hagan feliz siempre", "Si no eres feliz no puedes vivir, no puedes tener una vida con significado", entre otras afirmaciones trasmitidas, mantenidas y altamente valoradas culturalmente; y en esa lógica, los medios, a veces los profesionales, proporcionan diversos remedios, desde todo tipo de terapias psicológicas hasta tratamientos farmacológicos que, persiguiendo el mismo fin, pueden acabar convirtiéndose en un mal remedio de por vida, al limitar las actuaciones humanas posibles a unas pocas y no necesariamente particulares a esa persona que llega a consulta, sin considerar que tan valiosas son estas posibilidades y si apuntan a una vida con significado para ese individuo que ha de vivir esa vida día a día.

Lo cierto es que si una persona entra en esa espiral, es fácil que llegue a tener un funcionamiento rígido, poco flexible e incluso literalizado y desligado de las contingencias presentes en el contexto; este patrón de comportamiento que se circunscribe a evitar experiencias privadas que valora negativamente, facilita que la persona se aleje cada vez más de acciones en la dirección valiosa, en lo que realmente es importante para él. Cuando este patrón rígido, inflexible con sus propios eventos privados, se generaliza y cronifica, el resultado es una vida muy limitada para la persona que sufre, cada vez más y con mayor intensidad, por rechazar el malestar. Este tipo de sufrimiento es distinto del sufrimiento "normal" (en tanto derivado inevitablemente) que, a veces, acompaña al ser humano, en su intensidad, cronicidad y en el nivel de coerción que a la larga genera en la persona al tratar de removerlo de su vida, limitando sus opciones de decisión y por tanto limitando su vida en distintos contextos, situaciones, entre otros elementos donde pueden presentarse decisiones que para esta persona son importantes en su construcción de la vida con sentido que quiere y que le gustaría comprometerse a tener. Cuando esto ocurre hablamos de un patrón destructivo de evitación experiencial (o trastorno de evitación experiencial, TEE).

Tanto la ausencia como el exceso de la conducta gobernada por reglas han sido relevantes en la explicación de la depresión (Kanter et al., 2005). Para Rehm (1988), la depresión es resultado en la falla para desarrollar la conducta gobernada por reglas y la generalización de la auto-instrucción, por lo cual la persona puede verse demasiado controlada por las contingencias inmediatas y de esta manera, las metas a largo plazo y la planificación pueden verse afectadas.

Este autor predice que los individuos deprimidos pueden tener problemas relacionados con el auto-control, la formulación de auto-regulación en situaciones específicas y el abandono en la búsqueda de recompensas a consecuencias a largo plazo por la búsqueda de reforzamiento a corto plazo.

El modelo sugiere entonces, que las personas pueden deprimirse si se enfrentan repetidamente a situaciones donde las consecuencias se posponen o son impredecibles con respecto al resultado. En consecuencia, los individuos deprimidos tienen déficit en la habilidad de monitorear su propio comportamiento, además de una dificultad en generar reglas y soluciones a situaciones problemáticas. El déficit en la conducta gobernada por reglas de las personas deprimidas puede tratarse a través de un programa de intervención de auto-manejo cuyos objetivos según Rehm (1988) consisten en mejorar el auto-control, la auto-evaluación y el autoreforzamiento (Kanter et al., 2005).

La conducta gobernada por reglas excesiva y la evitación experiencial están igualmente relacionadas con la depresión. La evitación experiencial se define como el deseo por evitar el contacto con determinadas experiencias, seguido por intentos de escapar o evitar estas experiencias, aún cuando esta evitación cause daño psicológico. El repertorio de un individuo puede estar dominado por una evitación experiencial gobernada por reglas lo que puede conllevar a la depresión por diferentes razones. En primer lugar, la sobre dependencia a las reglas verbales previene a las contingencias ambientales directas de controlar el comportamiento. De esta manera, el estricto seguimiento de reglas puede conllevar a repertorios de conducta no efectivos que son insensibles a las contingencias cambiantes (Kanter et al., 2005).

Saunders, Peterson, Sampson y Reardon (2000) plantean que la depresión está usualmente asociada con dificultades de concentración y toma de decisiones, las cuales son esenciales en la efectiva solución de problemas y toma de decisiones en todas las facetas de la vida.

La inhabilidad de las personas deprimidas para realizar estas actividades tiene una gran importancia en la escogencia de una carrera. La indecisión en la escogencia de una carrera está relacionada con una identidad vocacional débil y pensamientos acerca de la carrera incapacitantes que dan paso a un alto estado de indecisión. Además se plantea que los estados afectivos asociados a la indecisión en la escogencia de una carrera como la depresión, ansiedad y un locus de control externo pueden asociarse con una identidad vocacional débil o a un pensamiento caótico y confuso. 
El aporte del análisis funcional en la compresión de la depresión a partir de las teorías conductuales es sumamente notable.

El análisis funcional constituye una herramienta básica para el estudio y modificación de la conducta. Algunos autores lo definen como la identificación de relaciones funcionales que sean relevantes y causales en los comportamientos particulares de un individuo, lo que permite que este análisis sea muy útil en la formulación de casos clínicos donde se integran las evaluaciones comportamentales del pre-tratamiento y/o las de durante el tratamiento del paciente (Virués-Ortega \& Haynes, 2005).

Bolling, Kohlberg y Chauncey (2000), en sus aportes del análisis del comportamiento en el estudio de la depresión, hacen referencia a la teoría triádica de la depresión de Beck, Rush y Emery (1979) que consiste en la visión negativa de sí mismo, del mundo y del futuro.

Los analistas del comportamiento conciben esta triada como repertorios conductuales que pueden ser producto de las experiencias (historia) de la persona y pueden conducir a la depresión; también afirman que la visión de sí mismo se desarrolla a partir de la interacción de la persona con su mundo social y que en las descripciones de sí mismo, se puede identificar claramente cuáles han sido los aspectos más importantes del individuo para los demás y que elementos durante la historia han sido seleccionados como importantes además de característicos de este individuo y que se ven reflejados en el constructo del sí mismo. Así, el sentido de sí mismo, resulta ser una función de la interacción social. Los autores mencionan que la interacción que se produce en la relación terapéutica tiende a formar contingencias que pueden influir en las descripciones que el paciente hace de sí mismo de forma mucho más breve que la terapia cognitiva estándar. En relación al segundo elemento de la triada -visión negativa del mundo, o tendencia del individuo para interpretar negativamente lo que le sucede-, los analistas conductuales plantean que esto tiene la función de evitar experiencias aversivas.

Es preciso resaltar que aunque las teorías mediacionales -cognitivas-y las no mediacionales -conductuales- pueden beneficiarse mutuamente, existen claras diferencias en sus interpretaciones y concepciones de la depresión, como se ha explicado en otros documentos (Novoa Gómez, 2004). Por ejemplo, el análisis funcional plantea que los pensamientos (conductas) negativos tienen una gran variedad de funciones, pero que no necesariamente son causales. Las estructuras cognitivas son entendidas como comportamientos moldeados por contingencias, los cuales pueden ser modificados a través de la experiencia con las contingencias.

$\mathrm{Al}$ trabajar con patrones de comportamiento moldeados por contingencias, puede hacerse una manipulación cognitiva directa con precaución por parte del terapeuta que apela a la argumentación lógica, principalmente a través de la construcción de reglas. Y cuando la respuesta de los pacientes a estas reglas se da por medio de la modificación de sus pensamientos, dicho cambio es conocido como comportamiento gobernado por reglas (Bolling et al., 2000).

Dentro de los modelos no mediacionales empleados en el tratamiento conductual podemos citar a la Teoría de Aceptación y Compromiso (ACT) utilizada en un gran número de casos clínicos con resultados exitosos. Por ejemplo, en un caso clínico de alcoholismo en el contexto de un estilo emocional dependiente de valoraciones, Luciano, Gómez, Hernández y Cabello (2001) aplicaron esta terapia. El tratamiento se orientó a romper aquellos contextos verbales que mantenían la evitación experiencial con ejemplos que fueron dirigidos a la posibilidad de detectar lo que es posible o no es posible cambiar, centrado en los costos y beneficios derivados de esto. Además, una parte importante de esta terapia, que orientó el proceso terapéutico de éste caso clínico, está relacionada con el interés por flexibilizar y desliteralizar los contextos verbales dominantes de la persona, lo cual se llevó a cabo mediante ejercicios, metáforas y paradojas que facilitan una toma de perspectiva más cercana a su realidad y que le permite al paciente tomar una decisión personal de manera responsable frente a su comportamiento, aceptando los eventos privados que conlleve ese proceder.

Además, es importante mencionar la psicoterapia analítico-funcional (PAF), la cual constituye una forma de terapia de conducta que hace especial énfasis en el uso de la relación terapeuta-cliente con el fin de utilizar con toda su intensidad las oportunidades de aprendizaje que surgen en la sesión terapéutica (por ejemplo, intervención in vivo). Según explican Kohlenberg, Tsai, Ferro, Valero, Fernández y Virués-Ortega, (2005) la PAF produce el cambio a través de contingencias de reforzamiento naturales y curativas que suceden dentro de una relación terapeuta-cliente emocional, cercana y con un alto grado de implicación.

En el ambiente terapéutico que se construye, los problemas cotidianos del cliente pueden ocurrir en sesión y ser modificados por las reacciones reforzantes naturales del terapeuta (Bolling et al., 2000). Un elemento importante de esta terapia que se debe considerar a la hora de su aplicación es que procura alterar las funciones de la "conducta clínicamente relevante" las cuales conforman apariciones en tiempo real durante la consulta de las problemáticas, mejorías o explicaciones del cliente, esto como medio para lograr cambiar las conductas reales del cliente, que son el foco del tratamiento. Este proceso se lleva a cabo esencialmente por medio del reforzamiento y la conducta verbal durante las sesiones.

Esta terapia emplea varias estrategias terapéuticas donde las tres principales son: a) Estar atento a la aparición de CCR, b) Provocar CCR, es decir reconstrucción de los problemas de conducta del cliente y c) Reforzar CCR2, fortalecimiento que el terapeuta ejerce sobre las mejorías que se producen en la consulta (Kohlberg et al., 2005). 


\section{Caso}

Se analiza el caso de FF, un hombre de 25 años de edad, soltero, sin hijos, con escolaridad bachiller, nacido el 14 de Septiembre de 1981 en la ciudad de Bogotá. Su nivel socioeconómico es medio bajo. A la fecha de inicio del proceso terapéutico se encontraba viviendo con su madre, su padrastro y su media hermana en la casa materna.

Genograma de la familia de FF:

FF acudió reportando insatisfacción "por mi forma de actuar, me dejo llevar por las cosas, me afectan mucho cosas que no me deberían afectar, soy muy sensible y todo me da ganas de llorar pero me controlo para no hacerlo... esta situación me hace sentir un dolor muy feo y no me gusta porque sé que es por cosas bobas" (noticias, novelas, películas...). Además refirió "quiero tener seguridad y organizar mi vida".

Describía episodios frecuentes e inusitados de llanto, labilidad emocional, hiperreactividad emocional cuando se encontraba solo, especialmente cuando estaba descansando, viendo TV o "sin hacer nada". FF no comprendía por qué le sucedía y refirió que "quería superarlo", consideraba que esto "era urgente". Según reportó, ésta situación afectaba a su familia, amigos y novia, porque había sido muy inestable a nivel académico, afectivo y laboral y creía que él era "el que predica pero no aplica". Decía que él "quería salir adelante, pero que no sabía cómo". Además, el paciente reportó que algunas veces (cada mes aproximadamente) tenía pesadillas de "cosas feas" que le podían pasar a las personas que quiere, como por ejemplo muertes.

Según el paciente estos patrones los ha tenido desde hace mucho tiempo, pero se acentuaron tras la finalización de la relación con su novia aproximadamente 1 año antes de la entrevista inicial, con quien llevaba varios años de noviazgo y había convivido durante 2 meses. FF toma la decisión de dejar la relación porque se sentía inseguro con respecto al futuro, en especial con la vida que les podría dar a sus hijos, "estaba muy confundido con respecto a qué hacer con mi vida". Después de que le sucedía esto, refería que pensaba qué podía estarle pasando y se preguntaba por qué tomaba esas reacciones frente a cosas que no eran importantes. FF reportaba que casi siempre presentía "qué le iba a pasar", en especial cuando estaba viendo televisión, y lo que hacía en ese momento era cambiar de canal y distraerse cambiando de actividad. Reportaba que él no se autorizaba a sí mismo llorar, trataba de detener el llanto porque cuando lloraba se sentía peor porque le daba rabia, mencionaba que con llorar no lograba nada.

\section{Descripción General}

FF trabaja como operario hace 2 años en una empresa. A pesar de haber iniciado estudios técnicos y universitarios, no ha terminado ninguna carrera. Después de graduarse del colegio empezó a estudiar en diferentes universidades, pero por problemas económicos y según reportaba dificultades en la comprensión de los temas, se tuvo que retirar. En la última ocasión se retiró porque su horario en el trabajo no le dejaba tiempo para estudiar, decía estar ascendiendo en la empresa y no quería dejar de trabajar porque para él era más importante su trabajo, por la estabilidad laboral, "estudiar es un sueño, no una meta".

Se describe como una persona callada, desde pequeño no se expresaba fácilmente a los demás, explicaba que este comportamiento pudo estar relacionado con el hecho de que su familia se trasteaba mucho y por lo tanto no permanecía por mucho tiempo en un mismo lugar, lo que dificultaba que pudiera conservar sus vínculos sociales. Para el paciente los problemas familiares lo han afectado desde niño "porque no podía hacer nada".

Mencionaba que una de las situaciones que tenía que vivir era el hecho de que la mamá tuviera muchos hombres "siempre tenía novios, pero ninguna relación le funcionaba". El núcleo familiar del paciente y con quienes vive está conformado por la mamá, el esposo de la mamá y una medio hermana menor. Sus padres están separados desde hace 20 años, "desde que él era pequeño". Tiene una hermana mayor. La mamá se volvió a casar con quien es su esposo actual $\mathrm{y}$ tuvo una niña que tiene 12 años de edad (con quien vive FF). Tiene otra medio hermana menor (13 años) por parte del papá, quien se volvió a casar. En cuanto a las relaciones con los miembros de la familia con quienes vive, describe a su mamá como una persona cariñosa, sobreprotectora y nerviosa. En el momento de la psicoterapia, su mamá estaba asistiendo al psicólogo porque "su estado de ánimo es depresivo y es muy nerviosa", ha estado en ínter consulta con psiquiatría y estaba medicada para poder conciliar el sueño. Con el esposo de la mamá FF tiene un trato cotidiano, pero no existe cariño ni respeto, según el paciente, esto es así porque "él la ha embarrado mucho con mi mamá". FF reportaba que con su padre no mantenía una relación constante y se hablaban ocasionalmente por teléfono, no lo hacía por iniciativa propia, sino porque su mamá se lo pedía. Su relación se trataba especialmente sobre temas de dinero y conversaciones muy superficiales, según FF "no existía relación padre-hijo".

Al inicio del proceso terapéutico reportaba que era nervioso con las personas que no conocía, y que en estas circunstancias sudaba o temblaba, y siempre le ocurría cuando estaba frente a un público. Por otra parte, reportó que nunca tuvo iniciativa de hacer las cosas, "he sido siempre muy apagado, con mi familia no he sido cariñoso, ahora estoy cambiando eso". En el colegio le iba muy bien y no se esforzaba en las materias, pero refiere que desde que se graduó no ha tenido buen desempeño en el estudio.

\section{Evaluación}

Entrevista clínica conductual con FF, entrevista clínica conductual con la novia, realización de auto informes, 
observación de conductas clínicamente relevantes y aplicación de los cuestionarios Índice de Bienestar Psicológico (Dupuy, 1984) y Escala Multidimensional de AsertividadEMA (Florez y Díaz-Loving, 2004).

La información obtenida por estos instrumentos mostró que en el momento en que acudió FF a consulta su bienestar psicológico era positivo aunque situado en los límites inferiores aproximándose a malestar moderado. Además, presentaba un predominio de no asertividad, lo cual representa a una persona inhábil para expresar deseos, opiniones y sentimientos que provoca conflictos en el individuo mismo $\mathrm{y}$ con las otras personas con las que se relaciona, se pierde seguridad, control de la situación, energía y eficiencia en la solución de problemas.

\section{La formulación del caso se realizó de la siguiente forma:}

FF presenta un patrón de conducta caracterizado por labilidad emocional, déficit en asertividad, desesperanza, unido a dificultades en toma de decisiones y en la persistencia conductual hacia la consecución de metas, todo lo cual deriva en una negativa y excesiva conducta auto referencial (pobre valoración de sí mismo). Este patrón fue adquirido por eventos incontrolables de desintegración familiar a corta edad, modelo de inestabilidad afectiva e hipervigilancia orgánica por parte de la madre como estrategia de control hacia terceros, estilo maternal sobreprotector y paternal ausente afectivamente. El patrón de conducta presentado por el consultante era mantenido por refuerzo social positivo limitado, reforzamiento negativo por evitación y escape ante las relaciones familiares constructivas, relaciones afectivas y con pares estables, sumado a la continua sobreprotección de la madre, así como al cumplimiento estricto de las reglas derivado de la poca sensibilidad a las contingencias directas. Se destaca la evitación de sus eventos privados como eje central del problema.

\section{Procedimiento}

La intervención fue llevada a cabo en consulta privada entre el psicólogo y el consultante, en sesiones de aproximadamente una hora de duración y con una frecuencia de 1 sesión semanal durante 12 semanas.

A partir del análisis funcional del caso, se realizó el plan de intervención tomando como focos claros de intervención las conductas descritas en la unidad de análisis. Se trabajó con el consultante sobre las conductas que le ocasionaban aislamiento y malestar psicológico, en contextos laborales, sociales y familiares, demostrando con hechos claros que las reglas establecidas por él no tenían una evidencia real en su vida.

Por medio de la confrontación y la búsqueda de hechos reales en el contexto del consultante, se buscó que él mismo derivara nuevas reglas sobre su conducta o modificara las que ya tenía. Además se identificaron los pensamientos,

Tabla 1. Esquema del Proceso terapéutico llevado a cabo con el caso de FF

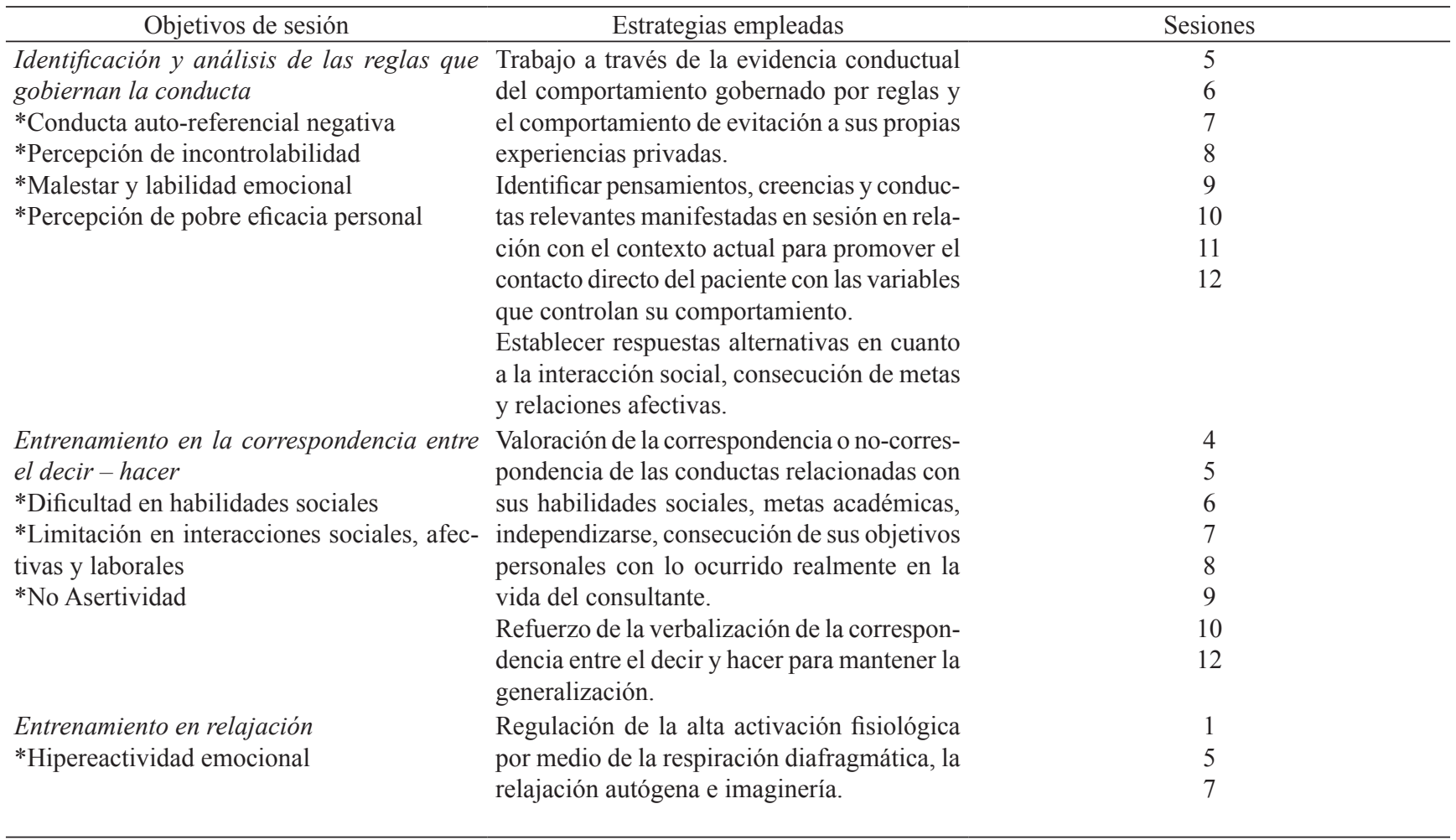


creencias y conductas relevantes manifestadas en sesión en relación con el contexto actual para promover el contacto directo del paciente con las variables que controlaban su comportamiento. Igualmente, se logró el establecimiento de respuestas alternativas por parte del paciente, en cuanto a la interacción social, consecución de metas, relaciones afectivas. Se realizó un entrenamiento en relajación para lograr la regulación de la alta activación fisiológica por medio de la respiración diafragmática, la relajación autógena e imaginería.

Y finalmente en cuanto a la dificultad en habilidades sociales se realizó un entrenamiento en la correspondencia entre el decir - hacer en relación con las habilidades sociales del paciente, sus metas académicas, independizarse, la toma de decisiones y consecución de sus objetivos personales y además se otorgó un refuerzo de la verbalización de la correspondencia entre el decir y hacer para mantener la generalización. El esquema general de la intervención se presenta en la tabla 1 .

\section{Resultados}

La efectividad de la intervención se ve reflejada en la grafica 1. En primer lugar se observa una disminución de la frecuencia de conductas auto referenciales negativas (se presenta un decremento importante a partir de la sesión 4, con un ligero incremento en la sesión 7 , pero se mantiene disminuido posteriormente); percepción de incontrolabilidad (se aprecia una continua disminución constante desde el inicio de las sesiones); malestar y labilidad emocional (se presenta un decremento desde la sesión 5 pero hay un aumento en las sesiones 6 y 7 a partir de un evento familiar estresante) y pobre autoeficacia (se presenta una disminución importante a partir de la sesión 2 con un ligero aumento durante las sesiones 5 a la 7, relacionadas con la ruptura de su relación amorosa con su novia, posteriormente no se vuelve a presentar esta conducta). Por otra parte, los resultados del Índice de Bienestar Psicológico (Dupuy, 1984) indican que hubo un cambio positivo en el bienestar psicológico del consultante desde la aplicación inicial ( 77 puntos) y la aplicación final (116 puntos), lo cual indica que al final del tratamiento, el bienestar psicológico del consultante era positivo a diferencia del inicial, el cual se encontraba en los límites inferiores, alcanzando un bienestar psicológico moderado.

En cuanto a la dificultad de las habilidades sociales, relacionadas con la comunicación poco asertiva y la limitación en las interacciones sociales, afectivas y laborales se observa una notable disminución, con un ligero rebote durante las sesiones 5 a la 7 ocasionado por el evento que experimentó el consultante (ruptura amorosa) mencionado en el párrafo anterior, que disminuye en las siguientes sesiones (Gráfica 2). Así mismo, los resultados de la Escala Multidimensional de Asertividad (Florez y Díaz-Loving, 2004) revelan un notorio cambio de los perfiles en la aplicación inicial y final (Tabla 2). Además de esto, en los reportes

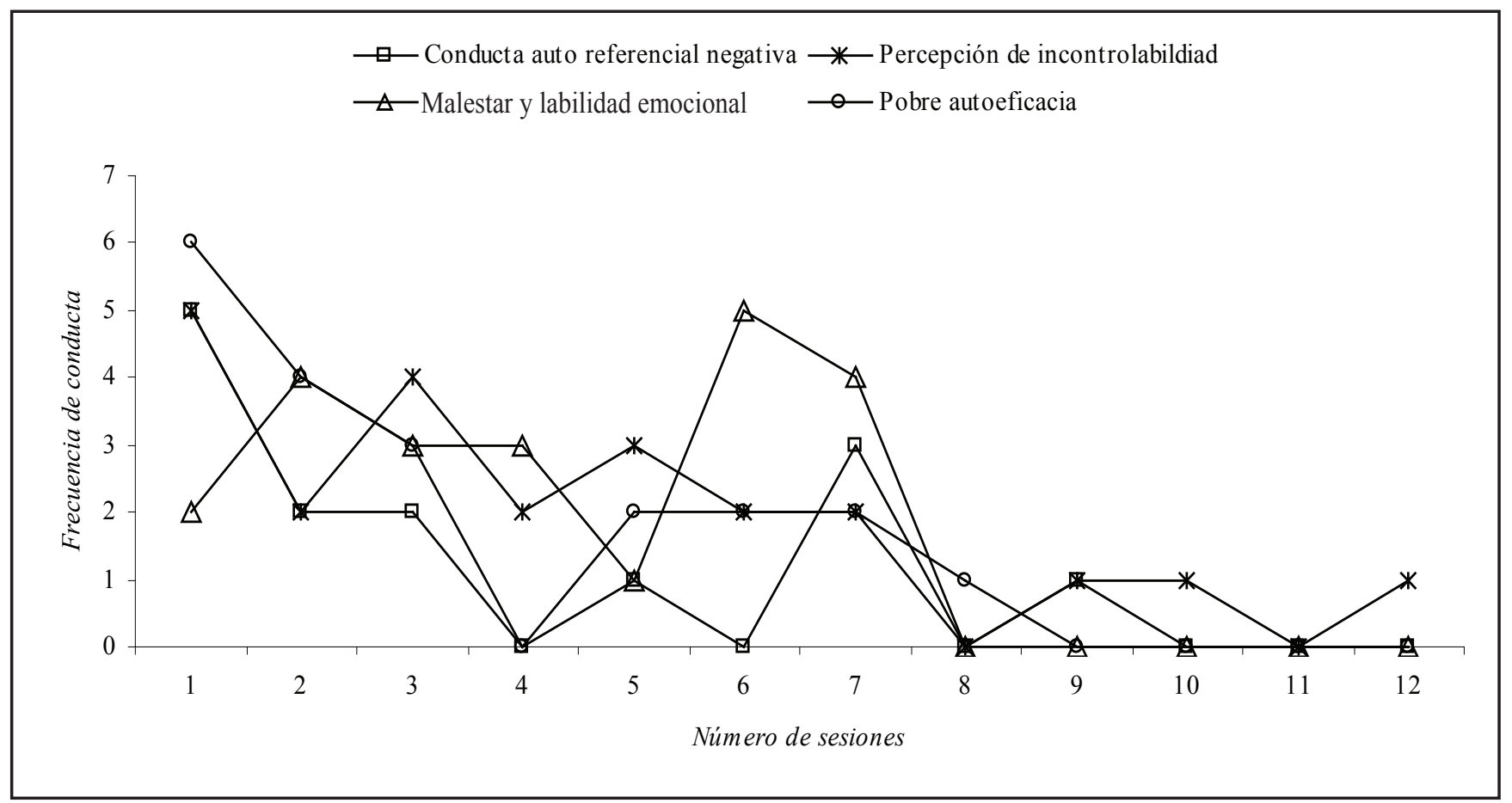

Figura 1. Evolución de las conductas auto referenciales negativas, malestar y labilidad emocional, percepción de incontrolabilidad y pobre autoeficacia. 
dados por el consultante durante las últimas sesiones de la psicoterapia, se debe resaltar que éste inició de nuevo una relación de noviazgo con su novia y reportó sentirse "feliz y tranquilo". Además, la red de apoyo de FF al iniciar las sesiones se conformaba solamente por su novia, según él mismo lo reportaba, pero al finalizar el proceso terapéutico refirió que cuenta con sus amigos, "pocos, pero muy buenos" y con sus compañeros del trabajo. Durante las sesiones finales de tratamiento, refirió que se encontraba mucho más cerca de su padre y se le facilitaba tener expresiones de cariño hacia él. En la última sesión de tratamiento refirió que quería estar pendiente de su papá porque considera que son buenos amigos y "ahora me nace ser cariñoso con él". Al finalizar la terapia se sentía más tranquilo para hablar con personas desconocidas o que representen autoridad, como por ejemplo su jefe. Aproximadamente en la séptima sesión reportó que las pesadillas ya no se presentaban porque se encontraba ocupado y hasta la última sesión no se volvieron a presentar.
En el caso de la hipereactividad emocional se evidencian algunas fluctuaciones durante las primeras sesiones, pero a partir de la sesión número 7, se presenta una disminución significativa que continúa hasta el final de las sesiones (Gráfica 3). Durante las sesiones finales de la terapia, FF reportó escasas situaciones de llanto y las que se reportaron fueron relacionadas con la situación problemática de la mamá y su esposo. Durante las sesiones iniciales, reportaba que hasta ahora estaba recapacitando sobre lo que había hecho antes y lo que quiere hacer ahora, porque consideraba que había cometido errores en su pasado, reportaba "puede ser que yo sea una persona de altibajos, a veces estoy bien y a veces estoy mal". Posteriormente, durante las sesiones finales del tratamiento, refería a este respecto "yo no he cometido errores en mi vida, entendí que simplemente he tomado decisiones y punto".

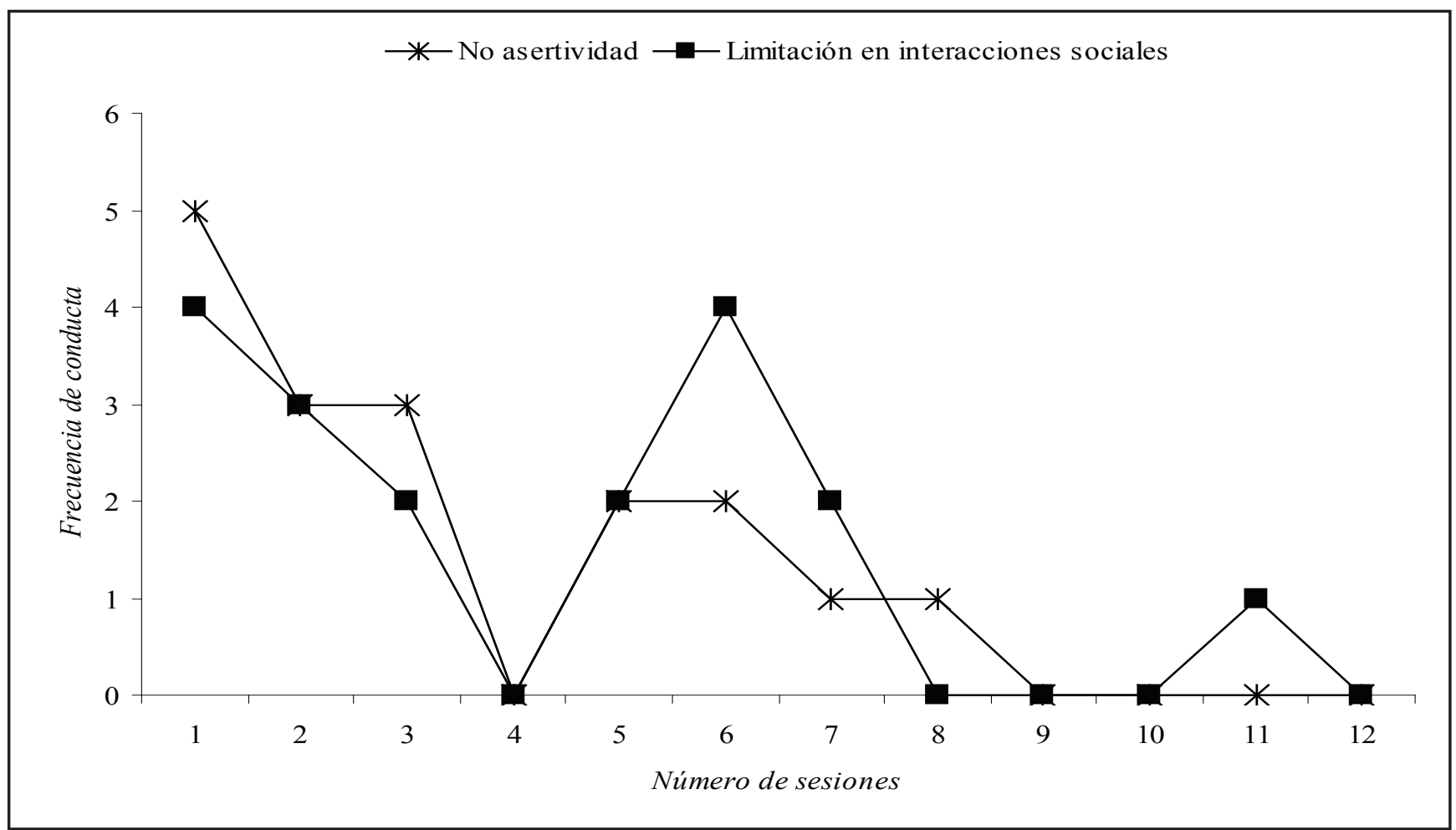

Figura 2. Evolución de las conductas relacionadas con la dificultad en interacciones sociales.

Tabla 2. Puntuación de los perfiles de la Escala Multidimensional de Asertividad (EMA) al inicio y final del tratamiento.

\begin{tabular}{|c|c|c|}
\hline Perfiles & Inicio tratamiento & Final tratamiento \\
\hline Asertividad indirecta & 44 & 30 \\
\hline No asertividad & 54 & 39 \\
\hline Asertividad & 50 & 62 \\
\hline
\end{tabular}




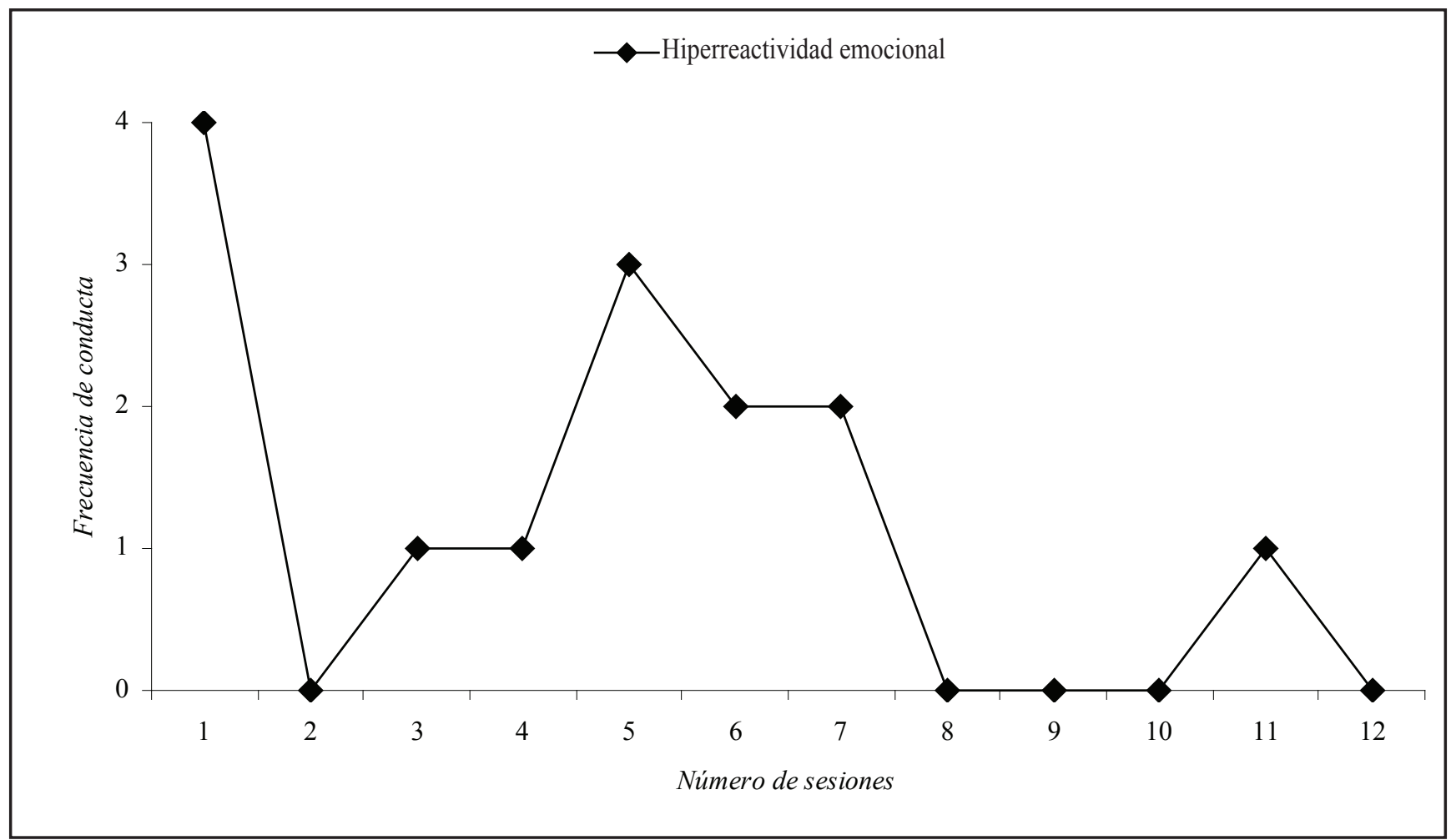

Figura 3. Evolución de las conductas de hipereactividad emocional.

\section{Discusión}

La intervención se realizó centrada en la formulación de caso, aplicando elementos derivados de la PAF y ACT y modificación del comportamiento gobernado por reglas, los cuales fueron descritos en el procedimiento del tratamiento.

Para la detección de los focos claves de la intervención fue esencial rescatar la importancia de la observación de las CCR presentadas durante las sesiones, las cuales constituyen un elemento muy valioso de la PAF.

Por otra parte, el estudio de este caso permitió comprobar la coherencia con algunos aportes teóricos citados inicialmente. En relación con los factores involucrados en la depresión planteados por Kanter et al. (2005), se encontró que el análisis del caso evidencia una estrecha relación con el primer factor, relacionado con la deprivación de reforzamiento.

Claramente, desde muy temprano en la historia de FF fueron evidentes varios principios conductuales como la pérdida de refuerzo, a partir de la disgregación familiar, erosión del reforzamiento, manifestada en una sobre protección materna y ausencia afectiva paterna y déficit en habilidades que le dificultó la consecución de sus metas a corto y largo plazo.

Además de esto, aunque Kanter et al. (2005), mencionan que los aportes sobre las alteraciones en el funcionamiento interpersonal pueden ser un producto de un déficit en las habilidades sociales y a su vez precipitantes y consecuentes de la depresión, no son un postulado que pueda dar una explicación completa a la depresión, los hallazgos de este caso pueden confirmar las consideraciones expuestas al interior de este modelo.

La dificultad en habilidades sociales de FF caracterizada por patrones de conducta específicos (detallados en dicho apartado), alteraron notablemente sus interacciones sociales a nivel familiar, laboral y afectivo, provocando así altos niveles de ansiedad con predominancia de hipereactividad fisiológica. Estos resultados analizados, señalarían que dicha dificultad en las habilidades sociales del paciente pudieron ser un desencadenante o precipitante de la conducta depresiva, según lo encontrado en las CCR3 durante las sesiones, así como de la definición de la unidad de análisis en este caso y que incluyo los componentes antes enunciados.

Contrario a lo que plantean Kanter et al. (2005), en el cuarto factor relacionado con el reforzamiento positivo del comportamiento depresivo, en el que establece una relación directa entre los comportamientos de malestar, como quejarse y el refuerzo de la comunidad social hacia estos, afirmándolo como causa del incremento de la conducta depresiva, el caso de FF no coincide con dicha afirmación. Su percepción de pobre eficacia personal y sentimiento de minusvalía nunca fue manifestado a su comunidad verbal, sino por el contrario, fueron conductas auto referenciales 
que igualmente tuvieron una influencia en el desarrollo de su conducta depresiva.

El cumplimiento estricto de las reglas del paciente se reflejó en la evitación experiencial por el seguimiento de reglas inexactas, relacionado con un tipo particular de regulación verbal, del grupo pliance (Dymond, Roche 6 Rehfeldt, 2005) (CGR), siendo sus pensamientos, creencias y conductas relevantes una influencia directa en las reglas que controlan su comportamiento. Esta formulación, encaja de manera precisa con lo planteado por Kanter et al. (2005), quienes sostienen que el repertorio de un individuo puede estar dominado por una evitación experiencial gobernada por reglas lo que puede conllevar a la depresión. Antes de la intervención FF sostenía dentro de sus creencias que él era "el que predica pero no aplica", frase que justificaba la mayoría de sus conductas auto referenciales negativas. Así mismo, la idealización de su pareja como la única mujer perfecta, creencia que fue haciéndose cada vez más fuerte, ocasionó que FF sufriera de manera excesiva por el rompimiento de su relación e impidió que FF pudiera iniciar posteriores relaciones afectivas con otras mujeres. En consecuencia, como lo plantean los autores, la sobre dependencia de las reglas verbales perjudicó que las contingencias ambientales directas controlaran su comportamiento, por lo tanto, se derivó en repertorios de conducta relacionados con la depresión que resultaron no efectivos.

Por otro lado, la Teoría de los Marcos Relacionales (TMR), puede dar mayores luces en la comprensión de la evitación experiencial del paciente y de algunas de sus conductas. La TMR es una teoría moderna del lenguaje y la cognición que hace gran énfasis en la trasformación de funciones de estímulo, teniendo en cuenta las múltiples relaciones de éste (Dymond, Roche \& Rehfeldt, 2005). Algunos autores hacen hincapié en que el sufrimiento humano ese encuentra estrechamente ligado con el lenguaje y al relacionar palabras, objetos, eventos y recuerdos, el individuo puede estar desencadenando sus problemas (Dymond, Roche \& Rehfeldt, 2005; Kohlenberg et al., 2005). Así la transformación de funciones y las relaciones de equivalencia que se presentan en el lenguaje y la cognición de las personas puede extender o mantener los comportamientos. Para el caso de FF, hay ciertos aportes de la TMR que se pueden vincular a su análisis.

A partir del análisis de los reportes de caso y el desarrollo de la terapia en sí, se puede concluir que el lenguaje y los pensamientos del paciente tuvieron una gran influencia en su condición afectiva, es decir, tenía un marco de relación sobreestereotipado sobre el cual se hicieron rupturas. En este sentido, existe una alta probabilidad de que su comportamiento se haya relacionado directamente con relaciones de equivalencia entre estímulos que se generaron en el transcurso de su vida y en medio de su contexto social y familiar. Por ejemplo, "llorar es ser débil, hace que se empeoren las cosas y no soluciona nada" y la excesiva ansiedad que presentaba frente a figuras de autoridad, "Ser jefe significa ser superior. Yo soy inferior, estoy por debajo, por lo tanto, él puede hacerme daño, humillarme, burlarse de mi.". Acercarse o hablarle a su jefe suponía para FF un riesgo de humillación que le generaba ansiedad, por esta razón, lo evitaba.

Por otra parte, las explicaciones que FF hacía acerca de su comportamiento, así como las reglas inexactas que se derivaron y llevaron a un seguimiento rígido de éstas, pudieron haber sido el producto de la transformación de funciones de muchos estímulos ambientales que FF experimentó en su niñez o juventud y que se insertaron de manera inadecuada y desligada del contexto en marcos de relación que guiaron su conducta y que originaron su trastorno afectivo, con las conductas especificas exhibidas por FF.

La terapia ACT es la psicoterapia clínica conductual que más ha desarrollado e incorporado la TMR en su conceptuación e intervención a distintos problemas, incluida la depresión, teniendo en cuenta que la respuesta relacional y la trasformación de funciones son un proceso central en la psicopatología humana (Dymond, Roche \& Rehfeldt, 2005). Por esta razón, los vínculos entre estos elementos conceptuales y empíricos no deben pasarse por alto, pues resultan muy valiosos a la hora de poder interpretar e intervenir en el patrón de evitación experiencial, por el cual un individuo que acude a terapia.

En este sentido y en concordancia con el caso clínico de alcoholismo con la aplicación de ACT de Luciano, Gómez, Hernández y Cabello (2001), la intervención se orientó a romper aquellos contextos verbales que mantenían la evitación experiencial con ejemplos que fueron dirigidos a la posibilidad de detectar lo que es posible o no es posible cambiar, centrado en los costos y beneficios. Igualmente, al trabajar en la modificación del comportamiento gobernado por reglas, se pretendió flexibilizar y desarmar el seguimiento estricto de las reglas del paciente, soportado por el moldeamiento por contingencias por parte del terapeuta que plantean Bolling et al. (2000), a través de la argumentación lógica y sustentada para la modificación o construcción de nuevas reglas más ajustadas al contexto y a lo que el paciente ha definido como valioso en su vida. Los resultados obtenidos después del tratamiento exhiben una notable disminución de las conductas auto referenciales negativas, malestar y labilidad emocional, percepción de incontrolabilidad y pobre autoeficacia, siendo evidencia y un reflejo importante de la eficacia de este componente de los modelos no mediacionales de corte contextual, como el trabajado con este caso.

Por otra parte, existe cierta relación entre los resultados del caso con los hallazgos de Rehm (1988), que sustentan que la depresión es resultado en la falla para desarrollar la conducta gobernada por reglas adecuada, por lo cual la persona puede verse demasiado controlada por las contingencias inmediatas y de esta manera, las metas a largo plazo y la planificación pueden verse afectadas. Los individuos deprimidos pueden tener problemas relacionados con el auto-control y la falta 
de búsqueda de recompensas a consecuencias a largo plazo por la búsqueda de reforzamiento a corto plazo. En el caso de FF evidenciamos claramente un deterioro en la consecución de sus metas tanto a corto como a largo plazo, relacionadas con su estudio y trabajo, al desarrollar reglas literalizadas que sustentaban la falta del planteamiento de metas y la concepción de un fracaso a priori sobre las que ya se había planteado. En este punto, siguiendo lo planteado por Saunders et al. (2000), la persona deprimida tiene dificultades en toma de decisiones y concentración, las cuales tienen una repercusión directa en la escogencia de una carrera que tienen que ver con una identidad vocacional débil y pensamientos acerca de la carrera incapacitantes que dan paso a un alto estado de indecisión.

Finalmente, aunque no es posible desconocer la influencia de factores externos en el desenlace del tratamiento, como los acontecimientos novedosos en la vida del paciente (trabajo, vida familiar, relación sentimental, etc.), indudablemente, los resultados obtenidos del caso de FF, son una clara muestra de la eficacia y vigencia de la aplicación de los modelos no mediacionales de corte contextual, específicamente componentes de ACT y PAF en el tratamiento de la depresión y que permiten nutrir las evidencias que a nivel mundial existen de su pertinencia y relevancia en el campo terapéutico. Igualmente es indispensable señalar que son escasos los reportes e investigaciones acerca de la aplicación de estas terapias a casos de depresión o trastornos afectivos; en consecuencia, este artículo representa un valioso aporte disciplinar y aplicado, que soporta aún más los avances en intervención y empíricos que suponen estos enfoques terapéuticos, teniendo en cuenta que sus resultados evidencian un notable mejoramiento, evolución positiva y mantenimiento de los cambios en las conductas que fueron intervenidas durante el proceso.

\section{Referencias}

Brown, E., Varghese, F. \& McEwen, B. (2004). Association of Depression with Medical Illness: Does Cortisol Play a Role?. Biological Psychiatry, 55, 1-9.

Burger, J. (1984) Desire for control, locus of control, and proneness to depression. Joumal of personality, 52 (1), 71-89

Dinan, T. (2001). Novel approaches to the treatment of depression by modulating the hypothalamic-pituitary-adrenal axis. Human Psychopharmacology, 16, 89-93.

Dougher (Ed.) (2000). Clinical Behavior Analysis: Theory, Research, and Treatment. Reno, NV: Context Press.

Dupuy, H.J. (1984). The psychological general well- being (PGWB) Index. En: Badía, X., Salamero, M. \& Alonso, J. (2002) La medida de la salud. Guía de escalas de medición en español. Edimac: Barcelona.

Dymond, S., Roche, B. \& Rehfeldt, R. (2005). Teoría de los marcos relacionales y la transformación de las funciones del estímulo. Revista Latinoamericana de Psicología, 37 (2), 291-303.

Flórez M.R. \& Díaz-Loving, R. (2004). Escala Multidimensional de Asertividad (EMA). Editorial manual Moderno S.A. de C.V.

Gómez, C., \& Roa, M. (2002). Tratamiento de una depresión causada por pérdida afectiva: caso único. Acción Psicológica, 2, 195-204.

Hayes, S. C., Barnes-Holmes, D., \& Roche, B. (Eds.). (2001). Relational Frame Theory: A Post-Skinnerian account of human language and cognition. New York: Plenum Press.
Kanner, A.D., Coyne, J.C., Schaefer, C. \& Lazarus, R.S. (1981). Comparison of two modes of stress measurement: Daily hassles and uplifts versus major life events. Journal of Behavioral Medicine, 4, 1-39. En: Kanter, J., Cautilli, J., Busch, A. \& Baruch, D. (2005). Toward a comprehensive functional analysis of depressive behavior: Five environmental factors and a possible sixth and seventh. The behavior Analyst Today, 6 (1), 65-81.

Kanter, J., Cautilli, J., Busch, A. \& Baruch, D. (2005). Toward a comprehensive functional analysis of depressive behavior: Five environmental factors and a possible sixth and seventh. The behavior Analyst Today, $6(1), 65-81$.

Kohlenberg, R., Tsai, M., Ferro, R., Valero, L., Fernández, A. \& ViruésOrtega, J. (2005). Psicoterapia Analítico-Funcional y Terapia de Aceptación y Compromiso: teoría, aplicaciones y continuidad con el análisis del comportamiento. International Journal of Clinical and Health Psychology, 5 (2), 349-371.

Lara, M. \& Klein, D. (1999). Psychosocial processes underlying the maintenance and persistence of depression: Implications for understanding chronic depression. Clinical Psychology Review, 19 (5), 553-570.

Luciano, M.C., Gómez, S., Hernández, M. \& Cabello, F. (2001). Alcoholismo, evitación experiencial y terapia de aceptación y compromiso (ACT). Análisis y Modificación de Conducta, 27 (113), 333-371.

Ministerio de la Protección Social Republica de Colombia (2003) Un panorama Nacional de la Salud y la Enfermedad mental en Colombia. Informe Preliminar.

Montes, C. (2004) La Depresión y su Etiologia: Una Patología Compleja. [En línea]. http://caibco.ucv.ve/caibco/CAIBCO/Vitae/VitaeDieciocho/ Articulos/Psiquiatria/ArchivosPDF/psiquiatria.pdf [2006, septiembre 6].

Novoa Gómez, M. M. (2004) Entendiendo "lo subjetivo" desde el análisis del comportamiento y propuestas terapéuticas. Revista Pensamiento Psicológico, 3, 59-80

Paykel, E.S. (Ed.). (1982). Handbook of affective disorders. New York: Guilford. En: Kanter, J., Cautilli, J., Busch, A. \& Baruch, D. (2005). Toward a comprehensive functional analysis of depressive behavior: Five environmental factors and a possible sixth and seventh. The behavior Analyst Today, 6 (1), 65-81.

Pérez-Gómez, M., Junqué, C., Mercader, J.M. \& Berenguer, J. (2000). Aplicaciones de la espectroscopía por resonancia magnética en el estudio de la patología cerebral. Revista de Neurología, 30 (2), 155-160.

Quiñones, R. \& Sandoval, M. (1996) Procesos Verbales y Desórdenes Depresivos: Marcos Relacionales y Seguimiento de Instrucciones. Suma Psicológica, 3 (2), 147-172.

Rehm, L.P. (1988)- Self-management and cognitive processes in depression. En L.B. (Ed.), Cognitive processes in depression. New York: Guilford. En: Kanter, J., Cautilli, J., Busch, A., \& Baruch, D. (2005). Toward a comprehensive functional analysis of depressive behavior: Five environmental factors and a possible sixth and seventh. The behavior Analyst Today, 6 (1), 65-81.

Saunders, D., Peterson, G., Sampson, J. \& Reardon, R. (2000). Relation of Depression and Dysfunctional Career Thinking to Career Indecision. Journal of Vocational Behavior 56, 288-298.

Scholten, A. (2003). Depresión. [En línea]. http:// www.thirdage.com/ ebsco/files [2006, septiembre 6]

Seligman, M., Abramson, L. \& Semmel, A. (1979). Depressive Attributional Style. Journal of Abnormal Psychology, 88 (3), 242-247.

Skinner, B.F. (1953). Science and Human Behavior. New York: The Macmillan Company.

Skinner, B. F. (1957/1975). La psicología en la comprensión de la enfermedad mental. En Registro acumulativo. Barcelona: Fontanella, S.A.

Skinner, B.F. (1966) The phylogeny and ontogeny of behavior. Science, 153, 1205-13.

Skinner, B.F. (1974) Sobre el Conductismo. Barcelona. Fontanella

Virués-Ortega, J. \& Haynes, S. (2005). Functional analysis in behavior therapy: Behavioral foundations and clinical application. International Journal of Clinical and Health Psychology, 5 (3), 567-587.

Wilson, K. \& Luciano M.C. (2002). Terapia de Aceptación y Compromiso $(A C T)$. Madrid: Pirámide. 